\title{
Use of Centroidal Voronoi Diagram to Find Optimal Gate Locations to Minimize Mold Filling Time in Resin Transfer Molding
}

\author{
J. Wang ${ }^{1,2, * \dagger}$, P. Simacek ${ }^{1,2}$ and S.G. Advani ${ }^{1,2}$ \\ ${ }^{1}$ Department of Mechanical Engineering, University of Delaware, Newark, DE 19716, U.S. \\ ${ }^{2}$ Center for Composite Materials, University of Delaware, Newark, DE 19716, U.S.
}

\begin{abstract}
In Resin Transfer Molding (RTM) processes, liquid resin is injected into a dry reinforcement structure to create a composite part within given time limits. To reduce the fill time, resin may be injected into the mold through multiple gates. The minimum number of gates and their locations needs to be determined. To reduce the number of scenarios to be simulated, an iterative method is implemented for multiple-gate injection optimization. The inlet nodes on the mesh surface are used to generate a Voronoi Diagram of the mold geometry. Then the optimal Centroidal Voronoi Diagram (CVD) of the mold surface is searched iteratively. It is shown that the generation points associated with the optimal CVD correspond with the gate locations that yield the shortest fill time. The results are compared with exhaustive search and genetic algorithms results to illustrate the efficiency and accuracy of CVD method.
\end{abstract}

Keywords: E. Liquid composite moulding; E. Resin transfer moulding; C. Process Simulation; C. Computational modeling

\section{Introduction}

\subsection{Liquid Composite Molding (LCM)}

In Liquid Composite Molding (LCM) process, the fiber preforms are stacked inside the mold cavity. Catalyzed resin is then introduced into it through openings in the mold walls until all the empty spaces between the fibers are filled with the resin. Once the resin cures, the part is de-

\footnotetext{
${ }^{*}$ Correspondence to: Jiayin Wang, University of Delaware, 126 Spencer Lab, Newark, DE 19716, USA

${ }^{\dagger}$ E-mail: jywang@udel.edu
} 
molded. Resin Transfer Molding (RTM) is one common LCM process that uses a rigid mold and resin is injected into the mold using positive pressure. RTM is widely used because it allows one to manufacture complex net-shaped parts with good structural properties and class " $\mathrm{A}$ " surface finish [1] and is addressed in this paper.

During the mold filling step, resin must fill all the empty regions between the fibers to produce a composite with no voids or defects. Voids or dry fiber regions are usually a result of resin not saturating the part before the resin gels. This may be due to resin reaching the vent prematurely before covering all the fibers, or a simple outcome of the infusion taking too long (resin gelling before it reaches the vent). The latter would be avoided if the mold filling time is within the resin gel time limit. To this end one must provide strategic infusion locations and sufficient driving pressure. An effective method for reducing fill time is to use multiple injection gates[2-5]. The gates may be opened either sequentially during the resin impregnation stage, or all the injection gates can be opened simultaneously to reduce the fill time[2].

Over the last two decades, many control volume finite element models have been developed to model the mold filling process [6-23]. Reasonably accurate flow pattern and pressure profile within the mold during the processing cycle can be predicted once the permeability of the fabric and viscosity of the resin along with the gate locations are specified. Vent locations can then be determined based on the predicted mold filling pattern.

A well tested Finite Element/Control Volume (FE/CV) package known as "Liquid Injection Molding Simulation" (LIMS) developed at University of Delaware $[6,15,16]$ is used as the mold filling simulation engine for this research to create filling pattern during LCM processing. LIMS can simulate 3D flow but as LCM is most commonly used to manufacture net shape shell structures, it is reasonable to mesh the part geometry with 2-D shell elements.

\subsection{Multiple gate injection optimization}

The ever-reducing computational times (it takes less than one minute to run one simulation with LIMS on a mesh with 5000 nodes) and the current levels of modeling automation suggest that one can let the computer simulation generate all possible infusion scenarios and declare the most optimal gate location which will fill the mold in least amount of time. As the location of a gate is specified by a single node in the FE mesh, the problem is de-facto discrete and the number of 
simulations required to find one optimal gate is limited to number of nodes in the mesh. For a coarse mesh, say with 5000 nodes, one would need to execute 5000 simulations to find a single gate location that will provide the minimum time to fill the mold. Each simulation may take about a minute on a modern workstation, resulting in the best gate for minimum fill time in few hours.

However, this brute force method is not practical when multiple gate locations are required to reduce fill time further. For example for two gates, one would need $1.25 \times 10^{7}(5000 \times$ 4999 /2) simulations to execute which will take months to complete. For $\mathrm{N}$ gates, the number of

simulations to be executed on a 5000 node mesh will be $C_{5000}^{N}$. Thus, using the brute force to find the best locations for more than one gate from all possible gate-location permutations is inefficient and impractical. Hence the need for an efficient optimization method which can find the most optimal multiple gate locations without resorting to millions of simulations. The objective of this paper is to construct models to calculate geodesic distances that account for part non-homogeneities, material variability and anisotropies. Then, the fill time cost functional is formulated and correlated with the modified geodesic distance model. Using this model, effective algorithms are further developed to reduce the computational times for multiple gate location optimization.

\section{Previous Work}

Previously, many local and global search techniques have been attempted to optimize gate location using mold filling simulations to replace exhaustive search methods [24-33]. Gradientbased methods (such as Quasi-Newton method) [29] have been used to optimize the gate locations for RTM. However, there is a danger that the searching process can converge to a local optimum[25]. Therefore, Genetic Algorithm (GA) and Artificial Neural Networks that are less prone to be trapped in a local optimum have been implemented to optimize gate locations in RTM to minimize cycle time [24,26-28,30-32]. However, the complexity in formulating performance index requires one to evaluate a large number of generations before it converges, and renders these method less effective to handle complicate geometries and material property. To improve the searching effectiveness, another meta-heuristic optimization method known as Branch and Bound Search (BBS) has been implemented to search for the optimum injection gate 
locations [25]. Compared with GA, BBS requires lower number of permutations to be evaluated. However, it is not clear how one can use this method to optimize multiple gate injections.

In this paper, we introduce modified Centroidal Voronoi Diagrams (CVD) [34] to search for the optimal multiple gate locations in RTM and demonstrate its utility for a variety of case studies with complex mold geometries. A one-dimensional model for mold flow is first developed and used to define the weighted geodesic distance and weighted fill time and their relations to each node on the mesh $[35,36]$. Next, this model is implemented on the spatial surfaces of the designed shell-like composite part to formulate a convex functional for fill time cost. Searching for the minimum of this functional is therefore used as the objective functional to determine the optimal gate locations. Next, the surface is re-interpreted using Voronoi Diagram [34] generated from the nodes that are set as gates on the mesh. Centroidal Voronoi Diagram (CVD), which is a specific type of VD of the surface, is proved to be the optimal solution that can minimize the cost functional [34]. However, this specific type of diagram of the surface is not readily available, and Lloyd's algorithm $[37,38]$ which is an iterative algorithm can be employed to search the CVD of the surface that minimizes the cost functional. The gate nodes identified in CVD are the optimal gate locations which will minimize the fill time. Finally, the efficiency and accuracy of CVD method is demonstrated by comparing the results with those returned by Exhaustive Search and Genetic Algorithm methods.

\section{Injection model and fill time functional definition for single gate injection}

Many models have been developed to correlate the fill time for each point on the part surface with its distance to the injection gate[30,31,36,39,40]. On flat surfaces, resin flow was assumed as the combination of radial flow on the surface and channel flow along the boundaries and resin time is formulated in terms of the Euclidian distance to the injection gate [39]. This model is further extended to polyhedral spaces[40]. However, material anisotropy and variability and more generalized complicated geometries were not accounted for in this approximate model. Therefore, another continuous one-dimensional model has been formulated to relate the fill time for each point with its distance to the injection gate based on Darcy's Law as shown in equation (1) [35]. 


$$
\int_{0}^{t} \frac{p_{i n}}{\eta} d t=\int_{0}^{L(t)} h(\psi) \cdot \phi(\psi) \cdot\left(\int_{0}^{\psi} \frac{d x}{K_{x x}(x) h(x)}\right) d \psi
$$

in which $p_{\text {in }}$ is the injection pressure, $\eta$ is resin viscosity, and $h, \phi, K$ are preform thickness, porosity and permeability respectively. Permeability variation in through thickness direction [41-43] is neglected and the porous domain is considered to be single scale. This model can be used to calculate fill time for each point on the surface based on its distance to the injection gate for 1D flow. In this paper, this model is discretized over 1D FE mesh, and further expanded to address spatial surface mesh of a complex domain based on the geodesic distance to the injection gate[31,36].

\subsection{Discretized correlations between fill time and distance to the gate}

First, equation (1) is discretized over the piecewise 1D FE mesh, which is shown in Figure 1. For an arbitrary node $i$ on the mesh, resin travels from node 0 to node $i$ sequentially. Fill time for node $i$, can then be calculated by integrating equation (1) from injection gate node 0 to node $i$, as is shown in equation (2):

$$
t_{i}=\sum_{j=1}^{i} \Delta t_{j}=\frac{\eta}{P_{\text {in }}} \sum_{j=1}^{i} \Delta x_{j} h_{j} \phi_{j}\left[\Sigma_{k=1}^{j-1}\left(\frac{\Delta x_{k}}{K_{k} h_{k}}\right)+\frac{\Delta x_{j}}{2 K_{j} h_{j}}\right], i=1,2 \ldots N
$$

Figure 1. One-dimensional flow model with mesh

The fill time, $t_{f}$ of a 1D part of length $L$ can be written as:

$$
t_{f}=\frac{\bar{\phi} \eta L^{2}}{2 \bar{K} P_{i n}}
$$

where permeability $\bar{K}$, thickness $\bar{h}$, and porosity $\bar{\phi}$, are the average material properties of the preform.

The variables in equation (2) are non-dimensionalized by 


$$
x^{*}=\frac{x}{L}, h^{*}=\frac{h}{L}, t^{*}=\frac{t}{t_{f}}, K^{*}=\frac{K}{K_{c}}, \phi^{*}=\frac{\phi}{\bar{\phi}}
$$

Thus, the non-dimensional form of equation (2) becomes:

$$
\begin{gathered}
t_{i}^{*}=\sum_{j=1}^{i} \Delta t_{j}^{*}=2 \sum_{j=1}^{i} \Delta x_{j}^{*} h_{j}^{*} \phi_{j}^{*}\left[\Sigma_{k=1}^{j-1}\left(\frac{\Delta x_{k}^{*}}{K_{k}^{*} h_{k}^{*}}\right)+\frac{\Delta x_{j}^{*}}{2 K_{j}^{*} h_{j}^{*}}\right] \\
=2 \sum_{j=1}^{i} K_{j}^{*} \phi_{j}^{*} h_{j}^{* 2} \cdot \frac{\Delta x_{j}^{*}}{K_{j}^{*} h_{j}^{*}}\left[\Sigma_{k=1}^{j-1}\left(\frac{\Delta x_{k}^{*}}{K_{k}^{*} h_{k}^{*}}\right)+\frac{\Delta x_{j}^{*}}{2 K_{j}^{*} h_{j}^{*}}\right], i=1,2 \ldots N
\end{gathered}
$$

Equation (5) includes the piecewise defined material variability over the mesh, and the elemental lengths are weighted by material permeability and thickness in the same pattern. Then we define the weighted fill time and weighted length for each element as:

$$
\begin{gathered}
\widetilde{\Delta t}_{J}^{*}:=\frac{\Delta t_{j}^{*}}{K_{j}^{*} \phi_{j}^{*} h_{j}^{* 2}} \\
{\widetilde{\Delta x_{j}}}^{*}:=\frac{\Delta x_{j}^{*}}{K_{j}^{*} h_{i}^{*}}
\end{gathered}
$$

and also define weighted fill time and weighted distance to the injection gate for each node as:

$$
\begin{aligned}
& \widetilde{t}_{l}^{*}:=\sum_{j=1}^{i} \widetilde{\Delta t}_{J}^{*} \\
& \tilde{x}^{*}:=\sum_{j=1}^{i} \widetilde{\Delta x}_{J}^{*}
\end{aligned}
$$

Equation (5) can then be written as:

$$
\widetilde{t_{l}^{*}}=\sum_{j=1}^{i} \widetilde{\Delta t}^{*}=2 \sum_{\mathrm{j}=1}^{\mathrm{i}}{\widetilde{\Delta x_{j}}}^{*} \sum_{k=1}^{j-1}{\widetilde{\Delta x_{k}}}^{*}+\sum_{j=1}^{i} \widetilde{\Delta x}^{*}=\left(\sum_{j=1}^{i} \widetilde{\Delta x}^{*}\right)^{2}={\widetilde{x_{l}}}^{* 2}
$$


This non-dimensional analysis provides a simple expression to relate each node's weighted fill time and its weighted distance to the injection gate.

\subsection{Geodesic distance definitions for the spatial surface}

For simplification, equation (6) - (8) are then implemented on spatial surfaces of any complex geometry by considering the surface mesh as a graph. Within each element, weighted resin travel time and length are defined using equation (6). The geodesic paths from the injection gate to any other nodes on the surface are calculated, and 1D resin flow is assumed along this path [36]. Thus, the distance between node $i$ on the part surface and the injection gate is the geodesic distance weighted by the material properties, and the weighted fill time for node $i$ can be calculated using equation (8). This allows one to define the first and second moments of the mesh with respect to the injection gate, and a cost functional can be formulated to calculate the cost in terms of time to fill the part. The objective of gate location optimization process is to minimize this fill time cost functional.

The weighted fill time and geodesic distance definition in equation (6) and equation (7) are then implemented on spatial surfaces. As is shown in Figure 2, resin is assumed to be travelling along the geodesic path which connects the injection gate with the current node $n$, and the weighted fill time and distance defined in equation (6) and equation (7) are used on each element on the resin travel path. Summation of all the $\widetilde{\Delta x}_{l}$ and $\widetilde{\Delta t}_{l}$ along the resin travel path will give the weighted geodesic distance connecting node $n$ and the injection gate and the weighted fill time of node $n$. Equation (8) is therefore further used to relate the weighted geodesic distance and weighted fill time for spatial surfaces.

Figure 2. 1D model (equation (8)) is further generalized for weighted geodesic distance and weighted fill time for node $n$ over a spatial surface

\subsection{First and second moments with respect to injection gate for spatial surfaces}

Based on the Control Volume definition (Figure 3) in Finite Element/Control Volume (FE/CV) solution schemes $[1,2,13,14]$ which has been used in mold filling simulation software LIMS, the weight function for each node is defined as: 


$$
w_{n}=\frac{V_{n}}{V_{m e s h}}
$$

in which $V_{n}$ is the control volume assigned for the node, and $V_{\text {mesh }}$ is the entire mesh volume. Obviously, the weight function satisfies:

$$
\sum_{n=\text { all nodes }} w_{n}=1
$$

Figure 3. Control volume assignment for each node in FE/CV approach [15,16,23].

Using the distance defined in equation (7), the first order of moment with respect to the injection gate can be written as

$$
\boldsymbol{M}=\sum_{n=\text { all nodes }} w_{n} \tilde{x}_{g \rightarrow n}^{*} \frac{\boldsymbol{r}_{\boldsymbol{n}}-\boldsymbol{r}_{\boldsymbol{g}}}{\left|\boldsymbol{r}_{\boldsymbol{n}}-\boldsymbol{r}_{\boldsymbol{g}}\right|}
$$

in which $w_{n}$ is the weight function for node $n$, and $\tilde{x}_{g \rightarrow n}^{*}$ is the weighted geodesic distance from injection gate to node $n$ using the distance definition in equation (7). For simplification, the coordinates of each node in the mesh is used to describe their position and calculate the direction vector for each node to the injection gate which is $\boldsymbol{r}_{\boldsymbol{n}}-\boldsymbol{r}_{\boldsymbol{g}}$ in equation (11). The flow centroid can be defined by setting $\boldsymbol{M}=0$, which yields the coordinates of the centroid:

$$
\boldsymbol{r}_{\boldsymbol{c}}=\left(c_{x}, c_{y}, c_{z}\right)=\left(\sum_{n=\text { all nodes }} w_{n} \tilde{x}_{g \rightarrow n}^{*} \boldsymbol{r}_{n}\right) /\left(\sum_{n=\text { all nodes }} w_{n} \tilde{x}_{g \rightarrow n}^{*}\right)
$$

In equation (12), the geodesic distance from the injection gate to node $n, \tilde{x}_{g \rightarrow n}^{*}$, is calculated using equation (8). However, to avoid the calculation of geodesic paths, weighted fill time $\widetilde{t}^{*}$ in equation (8) is substituted by the nodal fill time $t$ calculated by the flow simulation software, LIMS. Next, second moment tensor in global Cartesian Coordinates is defined as: 


$$
[I]=\left[\begin{array}{lll}
I_{x x} & I_{x y} & I_{x z} \\
I_{x y} & I_{y y} & I_{y z} \\
I_{x z} & I_{y z} & I_{z z}
\end{array}\right]
$$

Each component can be written as:

$$
\begin{aligned}
I_{x x} & =\sum_{n=\text { all nodes }} \frac{w_{n} t_{n}}{\left|\boldsymbol{r}_{\boldsymbol{n}}-\boldsymbol{r}_{\boldsymbol{c}}\right|^{2}}\left[\left(y_{n}-y_{c}\right)^{2}+\left(z_{n}-z_{c}\right)^{2}\right] \\
I_{y y} & =\sum_{n=\text { all nodes }} \frac{w_{n} t_{n}}{\left|\boldsymbol{r}_{\boldsymbol{n}}-\boldsymbol{r}_{\boldsymbol{c}}\right|^{2}}\left[\left(x_{n}-x_{c}\right)^{2}+\left(z_{n}-z_{c}\right)^{2}\right] \\
I_{z z} & =\sum_{n=\text { all nodes }} \frac{w_{n} t_{n}}{\left|\boldsymbol{r}_{\boldsymbol{n}}-\boldsymbol{r}_{\boldsymbol{c}}\right|^{2}}\left[\left(x_{n}-x_{c}\right)^{2}+\left(y_{n}-y_{c}\right)^{2}\right] \\
I_{x y} & =-\sum_{n=\text { all nodes }} \frac{w_{n} t_{n}}{\left|\boldsymbol{r}_{\boldsymbol{n}}-\boldsymbol{r}_{\boldsymbol{c}}\right|^{2}}\left[\left(x_{n}-x_{c}\right)\left(y_{n}-y_{c}\right)\right] \\
I_{x z} & =-\sum_{n=\text { all nodes }} \frac{w_{n} t_{n}}{\left|\boldsymbol{r}_{\boldsymbol{n}}-\boldsymbol{r}_{\boldsymbol{c}}\right|^{2}}\left[\left(x_{n}-x_{c}\right)\left(z_{n}-z_{c}\right)\right] \\
I_{y z} & =-\sum_{n=\text { all nodes }} \frac{w_{n} t_{n}}{\left|\boldsymbol{r}_{\boldsymbol{n}}-\boldsymbol{r}_{\boldsymbol{c}}\right|^{2}}\left[\left(y_{n}-y_{c}\right)\left(z_{n}-z_{c}\right)\right]
\end{aligned}
$$

in which $t_{n}$ is the nodal fill time calculated by LIMS, and $\boldsymbol{r}_{\boldsymbol{n}}=\left(x_{n}, y_{n}, z_{n}\right), \boldsymbol{r}_{\boldsymbol{c}}=\left(x_{c}, y_{c}, z_{c}\right)$ are coordinates for node $n$ and the centroid node respectively. The eigenvalues and eigenvectors of [I] can provide the three principal axes of second moment and their norms. When the centroid defined in equation (12) is not on the surface, it can be projected onto the surface following the directions of the principal axes of second moment.

\subsection{Fill time cost functional definition for single gate injection}

Next, the objective functional to minimize is defined in terms of the mold filling time for single gate injection cases. Instead of using the largest fill time as the optimized function, a fill time cost functional which includes the material permeability and thickness is defined as follows: 


$$
\begin{gathered}
F(g):=\frac{1}{2} \sum_{n=\text { all nodes }} w_{n} \tilde{t}^{*} g \rightarrow n \\
=\frac{1}{2} \sum_{n=\text { all nodes }} w_{n} \tilde{x}_{g \rightarrow n}^{2}
\end{gathered}
$$

in which $g$ is the node that is set as the gate, $w_{n}$ is the weight function for each node. In equation (15), equation (8) is used to change the fill time functional $\left(F(g) \sim \tilde{t}^{*}\right)$ into the second order

distance functional $\left(F(g) \sim \tilde{x}^{* 2}\right)$. This functional is convex over the surface [34], and therefore, the minimum of this functional is reached when setting the gradient of $F$ equal to $\mathbf{0}$, which can be written as:

$$
\nabla F=\sum_{n=\text { all nodes }} w_{n} \tilde{x}_{g \rightarrow n} \frac{\boldsymbol{r}_{\boldsymbol{n}}-\boldsymbol{r}_{\boldsymbol{g}}}{\left|\boldsymbol{r}_{\boldsymbol{n}}-\boldsymbol{r}_{\boldsymbol{g}}\right|}=\mathbf{0}
$$

Obviously, the centroid location defined in equation (12) is the single gate solution for equation (16). The optimal single gate solution is therefore to set the gate at the centroid. If the centroid location given by equation (12) does not lie on the surface, the centroid will be projected onto the surface along the principal axes of second moment calculated in equation (14).

\section{Voronoi Diagram (VD) and Centroidal Voronoi Diagram (CVD) for multiple gate injection locations}

After the relation between fill time and geodesic distance model is formulated in section 3 , Voronoi diagram can be defined by a finite set of locations on the surface to separate the part surface into subdomains. Each subdomain is associated with one location and consists of all points that are closer to that location than to any other of the preselected locations. The distance used can either be the Euclidian distance (Figure 4(a)), or it can be generalized to a convex function of the distance (Figure 4(b)).

Figure 4. Voronoi Diagram of a square surface using (a) Euclidian distance; and using (b) the distance defined in equation (7) 
When multiple gates are required for the injection, the part surface can be segregated into regions according to the weighted geodesic distance to these gates. A generalized Voronoi Diagram (VD) of the part surface is first defined based on the weighted distance defined in equation (6) and equation (7). Fill time functional defined in equation (15) for single gate injection is further generalized for multiple gate injection cases. The optimization objective for single gate to minimize the fill time cost is thus transformed into finding the optimal Voronoi Diagram of the part surface such that the fill time functional is minimum. Next, a special VD called as Centroidal Voronoi Diagram (CVD) of the part surface is defined based on the weighted fill time and weighted geodesic distance definition. It has been proven that the nodes set as generators for CVDs is the optimal solution for minimizing the fill time cost functional. Finally, an iterative algorithm, Lloyd's Algorithm, is used to search for the CVDs of part surfaces, which will give the optimal multiple gate locations' configuration that returns the minimum fill time cost [37].

\subsection{VD and CVD of the surface and fill time functional definition for multiple injection gates}

For a part with a total number of $k$ gates: $\left\{g_{i}\right\}_{i=1}^{k}$ the meshed surface of the part is separated into $k$ regions: $\left\{V_{i}\right\}_{i=1}^{k}$, each of which is called a Voronoi Diagram (VD) of the part and is associated with each gate accordingly. Each node contained in a specific VD $V_{i}$ corresponding to the gate $g_{i}$ satisfies the following equation:

$$
V_{i}=\left\{n \in \Omega \mid \tilde{x}^{*}{ }_{g_{i} \rightarrow n}<\tilde{x}^{*}{ }_{g_{j} \rightarrow n} \text { for } j=1, \ldots, k, j \neq i\right\}
$$

In equation (17), $\Omega$ is the set for all the nodes in the mesh, and $\tilde{x}^{*} g_{i \rightarrow n}$ is the geodesic distance from gate $g_{i}$ to node $n$, which is defined in equation (7). Because equation (8) describes a simple monotonous (quadratic) relations between (non-negative) weighted fill time $\tilde{t}^{*}$ and geodesic distance $\tilde{x}^{*}$, the separation of the surface into regions based on the distance to the injection gates defined in equation (17) can be replaced with the following rule:

$$
V_{i}=\left\{n \in \Omega \mid \tilde{t}_{g_{i} \rightarrow n}<\tilde{t}_{g_{j} \rightarrow n}^{*} \text { for } j=1, \ldots, k, j \neq i\right\}
$$


The weighted fill time can be calculated from mold filling simulation using LIMS which provides the fill time for each node. Flow pattern contour can be plotted based on the fill time results, and the surface is separated into sub-regions based on the resin traces from each injection gate. An example of separating a square surface into four sub-regions based on the flow pattern using four-gate injection plan is shown in Figure 5.

Figure 5. Flow pattern for a square part surface when resin is introduced from four injection gates, and four Voronoi Diagrams are generated based on the fill time results.

Next, the fill time cost functional defined in equation (15) for single gate injection is further generalized for the injection scheme with a number of $k(k>1)$ gates $\left\{g_{i}\right\}_{i=1}^{k}$, which is as follows:

$$
F\left(\left\{V_{i}\right\}_{i=1}^{k},\left\{g_{i}\right\}_{i=1}^{k}\right)=\sum_{i=1}^{k} \sum_{n \in\left\{V_{i}\right\}} w_{n} \tilde{t}^{*} g_{i} \rightarrow n
$$

Next, the centroid for each Voronoi region $V_{i}$ associated with gate $g_{i}$ can be defined by:

$$
\begin{gathered}
\boldsymbol{M}_{\boldsymbol{i}}=\sum_{n \in\left\{V_{i}\right\}} w_{n} \tilde{x}_{g_{i} \rightarrow n}^{*} \frac{\boldsymbol{r}_{\boldsymbol{n}}-\boldsymbol{r}_{\boldsymbol{g}_{\boldsymbol{i}}}}{\left|\boldsymbol{r}_{\boldsymbol{n}}-\boldsymbol{r}_{\boldsymbol{g}_{\boldsymbol{i}} \mid}\right|} 0 \\
\boldsymbol{r}_{\boldsymbol{c} \boldsymbol{i}}=\left(c_{x i}, c_{y i}, c_{z i}\right)=\left(\sum_{n \in\left[V_{i}\right\}} w_{n} \tilde{x}_{g \rightarrow n}^{*} \boldsymbol{r}_{n}\right) /\left(\sum_{n \in\left\{V_{i}\right\}} w_{n} \tilde{x}_{g \rightarrow n}^{*}\right)
\end{gathered}
$$

For simplicity, the weighted geodesic distance from gate $g_{i}$ to node $n, \tilde{x}^{*} g_{i} \rightarrow n$, in equation (20) can be calculated from equation (8), in which the weighted fill time is substituted by the fill time returned by LIMS. If the centroid location calculated from equation (20) is not on the surface, it is projected onto the surface based on the three principal axes of second moment of the subregion according to the centroid $\boldsymbol{M}_{\boldsymbol{i}}$ using equation (14) over the sub-region.

Each multiple-gate pattern corresponds to a specific Voronoi Diagram of the surface. There exists a special Voronoi Diagram of the surface which is called Centroidal Voronoi Diagram (CVD). In CVD, the centroid of each sub-region coincides with the generator (gate) of that 
region (Figure 6). It has been proven that under CVD, the fill time cost functional defined in equation (19) reaches minimum, and therefore it provides the optimal multiple gate locations for the part [37]. Vent locations are then placed where the resin arrives last and where flow front merge based on the flow pattern return by LIMS in the last iteration. However, for some complicated geometries, one may not be able to place a vent at suggested locations. Besides, because the mathematical model is based on geodesic distance, the geometry of each Voronoi Diagram is required to be convex in geometry so that the CVD represents the optimal results. Also, CVD is usually not readily available and algorithms have been developed to search for a CVD of the surface.

Figure 6. Comparison between regular VD (left) and CVD (right) of the square surface with two injection gates

\subsection{Alternative way to calculate the flow centroid on spatial surfaces}

To loosen the convex shape requirement condition for each Voronoi Diagram, instead of using equation (20) to calculate the flow centroid for each Voronoi Diagram, we proposed an effective yet accurate method to locate the flow centroid through running LIMS by setting infusion lines on the outer boundary of each VD. To find the centroid for a specific Voronoi Diagram $V_{i}$, the boundary nodes of $V_{i}$ are first selected as infusion line, as shown in Figure 7 (a). All the nodes not contained by $V_{i}$ are set as filled. Next, LIMS is executed to calculate the flow pattern, and the last fill node is then set as the flow centroid (Figure 7(b)). This method executes one LIMS simulation to find the centroid for each Voronoi Diagram. This approach is numerically efficient and convenient as compared to calculating the geodesic distances using equation (20). An added advantage of using LIMS simulation is that it can be used to find centroid for VDs that contain non-homogeneous permeability regions. For example in Figure 8(a), the compaction around the heel section of the orthotic foot results in low permeability region. The centroid found for the Voronoi Diagram $V_{1}$, which is the same with $V_{1}$ in Figure 7, is moved up as shown in Figure 8(b). Using this method to find centroid for each Voronoi Diagram no longer requires that each Voronoi be geometrically convex. However, a much looser constraint that the flow pattern returned from each LIMS simulation has to be convex still needs to be guaranteed to achieve an accurate gate location optimization procedure. 
Figure 7. Search for the centroid of a Voronoi Diagram through running LIMS for homogeneous part: (a) Voronoi Diagram generation for three-gate injection of an orthotic foot $(b)$ flow centroid found for $V_{1}$ by running infusion simulation by setting infusion lines on the region boundary

Figure 8. Search for the centroid of a Voronoi Diagram for non-homogeneous part: (a) low permeability section at the heel due to preform compaction; (b) centroid found for Voronoi Diagram $V_{1}$.

\subsection{Lloyd's algorithm}

A well-constructed iterative algorithm, Lloyd's method, is used as the search algorithm to find the CVDs for a surface. It has been proven that Lloyd's algorithm will converge to the final optimal configuration in limited number of iterations $[37,38]$. Lloyd's algorithm is then combined with LIMS to accommodate multiple gate location optimization during mold filling. If the number of gates selected was $k$, the $k$-gates optimization in RTM is carried out using the modified Lloyd's algorithm as shown in Figure 9.

Figure 9. Flow chart that optimizes the location of $k$ gates using Lloyd's algorithm

For the $k$-gates optimization problem $(k>1)$ shown in Figure 9, one LIMS simulation is used to calculate each Voronoi Diagram region of the part surface, and " $k$ " simulation results are used to provide the centroid for each VD using the method introduced in section 4.2. Therefore the total number of simulations to find CVD of the part surface using modified Lloyd's algorithm is thus equal to (number of iterations) $\times(k+1)$. In this paper, CVD method is specifically referred as this searching method that employs modified Lloyd's algorithm in conjunction with LIMS to find the optimal $k$-injection locations (gates) in RTM. An example that uses the modified Lloyd's algorithm to find the optimal four-gate injection plan for a square part is shown in Figure 10.

Figure 10. Use of Lloyd's algorithm to find the optimal four-gate injection plan for a square surface. 
Comparing to the exhaustive method which quickly gets out of hand when number of gates are greater than two, Lloyd's algorithm can greatly reduce the number of scenarios to be executed in LIMS.

\section{Case studies}

In this section, case studies are shown to exemplify the implementation of CVD method on multiple gate injection optimization. Examples chosen represent a wide range of composite parts that have manufacturing complexity in both geometry and material properties.

\subsection{Simple Square}

For comparisons, three sets of different initial four-gate locations are randomly selected for the square surface which uses isotropic and homogeneous fabric with permeability equal to $K=$ $1.0 \times 10^{-11} \mathrm{~m}^{2}$. As shown in Figure 11, all of the initial configurations converge to the final optimal gate within limited number of iterations, and the error of the results (distance between each of the four gates) are within two elemental lengths.

Figure 11. Different initial four-gate configurations for the square converge to the same optimal gate locations.

The Lloyd's algorithm may lead to local optimum instead of the global minimum of the fill time functional. An example of the iteration stopping at local optimum for the square surface is shown in Figure 12. However, because each sets of iteration requires limited number of simulations, this problem can be avoided by selecting different sets of initial gate configurations without adding significant computational effort.

Figure 12. An example of four-gate location optimization process converging to the local optimum for the square geometry.

The Lloyd's algorithm is then used for three-gate location optimization when anisotropic fabric is used or if the local permeabilities are modified due to the draping process [44]. The fabric permeability in the two principal directions are assigned as $K_{1}=2.0 \times 10^{-11} \mathrm{~m}^{2}$ and $K_{2}=1.0 \times$ $10^{-11} \mathrm{~m}^{2}$, respectively. Optimal three-gate locations are shown in Figure 13(a) for the $0^{\circ}$ laminate after 6 iterations and 24 simulations and the minimum fill time is 2125 s. Figure 13(b) 
shows the optimal three-gate locations for $-45^{\circ}$ laminates, and returns a minimum fill time of 2271s after 5 iterations and 20 simulations. Then CVD method is implemented to find the optimal gate locations when higher anisotropies $\left(K_{1}=10 K_{2}\right)$ are present (Figure $\left.13(\mathrm{c}),(\mathrm{d})\right)$. It can be shown that the optimal gate locations change with the laminate directions, and the CVD method converges and can well capture the flow behavior of anisotropic fabrics.

Figure 13. Three-gate optimization for the square with anisotropic fabric: (a) optimal gate locations for $0^{\circ}$ laminates after 6 iterations (24 simulations $\left.K_{1}=2 K_{2}:\right)$; (b) optimal gate locations for $-45^{\circ}$ laminates after 5 iterations $\left(20\right.$ simulations $\left.K_{1}=2 K_{2}\right)$; (c) optimal gate locations for $0^{\circ}$ laminates after 4 iterations (16 simulations $\left.K_{1}=10 K_{2}:\right)$; (d) optimal gate locations for $-45^{\circ}$ laminates after 4iterations (16 simulations $K_{1}=10 K_{2}$ )

\subsection{Orthotic leg geometry using isotropic material}

The fabric material used is isotropic with permeability $\mathrm{K}=1.92 \times 10^{-11} \mathrm{~m}^{2}$. The gate locations optimization, for a personalized orthotic leg device ( 15 inches tall) which has complex geometrical features, is important as the mold filling needs to be completed in less than 20 minutes. This is because the mold is injected with fast-cure resin systems and the mold filling process needs to be complete before the resin starts to gel and increase the resin viscosity exponentially restricting resin movement. Also, this becomes a bigger challenge as the injection pressure is limited to one atmosphere as the mold material is not designed to bear higher pressures without resulting in dimensional changes. Therefore the number of minimum injection gates and their locations have to be optimized so that the fill time is minimum and satisfies the cycle time requirement. Next, the CVD method is compared with Genetic Algorithm results and exhaustive search method.

\subsubsection{Minimum number of gates optimization for the fast cure resin injection}

As is shown in Figure 14, the centroid of the part is first calculated and projected onto the part surface and is set as the injection gate, which will give the shortest single gate injection plan. However, the shortest single gate fill time is 4468s which far exceeds the resin gel time of 1200s. Next, the CVD method is used to find optimal two-gate injection plan for this orthotic leg (Figure 15). In 4 iterations, the optimal two-gate injection plan is found and the shortest cycle time using two gates is $1506 \mathrm{~s}$, which is still more than resin gelation initiation time of $1200 \mathrm{~s}$. 
Next, three-gate injection plan is explored using the CVD method and a shortest fill time of 918s is reached (Figure 16), which is much below the gelation time and is completed by executing a total of 28 simulations. In comparison, the brute force for this part mesh which contains 4684 nodes is impossible because of the large number of simulations $\left(C_{4684}^{1}+C_{4684}^{2}+C_{4684}^{3}=\right.$ $1.71 \times 10^{10}$ ) needed.

Figure 14. Orthotic part design and optimal one-gate injection fill time result

Figure 15. Two-gate location optimization for the orthotic leg

Figure 16. Three-gate location optimization for the orthotic leg which satisfies the resin gel time restrictions

\subsubsection{Comparison of two-gate injection optimization using exhaustive search and Genetic Algorithm}

First, the two-gate locations for the orthotic part using CVD method is compared with the result returned using exhaustive method by listing all combinations of two-gate injection scenarios. Because the number of scenarios $\left(C_{4684}^{2}=11\right.$ million $)$ for this mesh is too large, the part is remeshed using a larger element size. The coarse mesh (Figure 17 (a)) has 210 nodes and the number of scenarios to be simulated in LIMS is $C_{210}^{2}=21945$. Because the coarse mesh cannot return the accurate fill time, the location of the gate found using exhaustive search method is then used on the fine mesh, whose nodes that are closest to the locations are set as gates and therefore yield the fill time in Figure 17 (a). Next, Genetic Algorithm is used on the fine mesh to find the two-gate injection locations of the optimal two-gate injection locations after 190 simulations with a batch size of 10 (Figure 17 (b)). Comparison of number of simulations and minimum fill time returned for the three methods are listed in Table 1. Because LIMS simulations requires the most calculation time in each iteration (in the order of $N^{2}$, in which $N$ is number of nodes in the mesh), the time cost for multiple gate optimization is represented using number of simulations in here.

Figure 17. Two-gate injection optimization results comparison when using (a): exhaustive search on the coarse mesh with 210 nodes, (b): Genetic Algorithm after 190 simulations, and (c): CVD method after 12 simulations. 
Table 1. Two-gate injection optimization comparison using different methods

\begin{tabular}{|c|c|c|c|}
\hline Optimization Method & $\begin{array}{c}\text { Exhaustive Search } \\
\text { (coarse mesh) }\end{array}$ & $\begin{array}{c}\text { GAs } \\
\text { (fine mesh) }\end{array}$ & $\begin{array}{c}\text { CVD } \\
\text { (fine mesh) }\end{array}$ \\
\hline Minimum Fill Time(s) & 1458 & 1481 & 1506 \\
\hline Number of Simulations & 21,945 & 190 & 12 (4 iterations) \\
\hline
\end{tabular}

\subsubsection{Comparison of three-gate injection optimization using Genetic Algorithm}

The CVD results for three-gate injection optimization are compared with that returned from Genetic Algorithm (GA) which is implemented on the fine mesh. GA results were obtained after 23 generations with a batch size of 10 . The total number of simulations required is therefore 230 . As shown in Figure 18, $g_{1}$ and $g_{2}$ found by both methods are at the same locations, and the gate $g_{3}$ on the foot of the orthotic leg are close enough to ensure a shortest fill time found with a $1.8 \%$ difference after 28 simulations.

Figure 18. Three-gate injection optimization results comparison when using (a): Genetic Algorithm after 230 simulations, and (b) CVD method after 28 simulations.

\subsection{Chassis}

The CVD method is then used on a chassis when both homogeneous material is used as shown in Figure 19 (a) and when insert is added which results in low permeable region as shown in Figure 19 (c). The two-gate location for homogeneous part is evenly distributed along the center line as shown in Figure 19 (b). Gate $g_{2}$ is a little off the center line because of the notch at the left bottom of the part. The two-gate location results when the insert is added in the lower part of the chassis is shown in Figure 19(d). The low permeable section over the insert has moved both the gates into the lower part of the chassis.

Figure 19. Two gate optimization for a chassis: (a) part geometry with homogeneous isotropic permeability, (b) optimal two-gate results after 15 simulations, (c) part geometry with low permeability region, (d) optimal two-gate results after 18 simulations. 
However, as mentioned in section 4.2, the flow pattern of the part has to be convex to use the CVD method. If the flow pattern in any of the iterations is non-convex, the results returned can deviate from optimal gate locations. For example in Figure 20, high permeable region occurs along the corner in lower right of the chassis, because of the gap between preform and the mold surface. When calculating the centroid for the Voronoi Diagram $V_{1}$ (Figure 20(b)) associated with $g_{1}$ by selecting the boundary of $V_{1}$ as infusion line, the flow pattern has two converging points, one of which is the last fill point, and the other is the point where flow front meets before the region is fully filled. Selecting the last fill point as the centroid which is shown in Figure 20 (c) is not accurate, because the extra flow converging point is ignored. The doubly connected flow pattern makes the CVD method inaccurate in placing the gate at the locations which will result in least time to fill.

Figure 20. Two gate location optimization for the chassis with flow disturbance (high permeability) region shown in (a), and (b) VDs calculation in iteration 5, and (c) inaccurate centroid found for $V_{1}$ because of the extra flow front meeting point.

\section{Summary and conclusions}

A one-dimensional model is first developed and discretized using finite elements. Weighted geodesic distance and weighted fill time and their relations to each node is defined based on this one-dimensional model. The weighted geodesic distance and weighted fill time is further generalized for spatial surfaces by considering the surface mesh as a graph. A fill time cost functional is defined for the surface mesh based on the weighted distance and fill time for each node, and the searching for the minimum of the fill time cost functional is used as the objective function to optimize gate locations. Next, the nodes that are set as gates are used as generators to create the Voronoi Diagram of the part surface, from which the optimal Centroidal Voronoi Diagram of the mold part surface, which will minimize the fill time cost functional, is found using iterative Lloyd's algorithm. It has been shown that the CVD of the part surface will return the gate locations that minimize the fill time cost functional, and requires fewer simulations to be run in LIMS compared to the exhaustive search method and Genetic Algorithms. Although trapping in the local optimum is observed for highly symmetrical geometries using homogeneous and isotropic material when selecting the initial gate location symmetrically, this scenario is 
highly unlikely for asymmetrical parts with material randomness, and can be avoided by setting random initial gate configurations. Vents are then placed at the places where resin arrives last and the location where the flow fronts meet for the optimal injection scheme from the mold filling pattern returned by LIMS to avoid air pockets being trapped. In this paper, fill time cost functional is considered as the only optimization objective and the number of vents and vent locations do not need to be optimized in our method to achieve minimal fill time.

\section{Acknowledgements}

Research was sponsored by the Army Research Laboratory and the Defense Advanced Research Projects Agency and was accomplished under Cooperative Agreement Number W911NF-13-20027.

The views and conclusions contained in this document are those of the authors and should not be interpreted as representing the official policies, either expressed or implied, of the Army Research Laboratory or the U.S. Government. The U.S. Government is authorized to reproduce and distribute reprints for Government purposes notwithstanding any copyright notation herein.

Input from Dr. Yarlagadda and Dr. Tierney at the University of Delaware, Center for Composite of Materials on the use of the Orthotic geometry and constraints associated with manufacturing allowed us to conduct a realistic simulation.

\section{References:}

[1] Advani SG, Sozer EM. Process modeling in composites manufacturing. CRC Press; 2012.

[2] Kang MK, Jung JJ, Lee W Il. Analysis of resin transfer moulding process with controlled multiple gates resin injection. Compos Part A Appl Sci Manuf 2000;31:407-22. doi:10.1016/S1359-835X(99)00086-X.

[3] Chan AW, Morgan RJ. Sequential multiple port injection for resin transfer molding of polymer composites. SAMPE Q 1992;24:45-9.

[4] Bickerton S, Stadtfeld HC, Steiner K V, Advani SG. Design and application of actively controlled injection schemes for resin-transfer molding. Compos Sci Technol 2001;61:1625-37. doi:10.1016/S0266-3538(01)00064-1.

[5] Berker B, Barooah P, Sun JQ. Sequential logic control of liquid injection molding with automatic vents and vent-to-gate converters. J Mater Process Manuf Sci 1997;6:81-104.

[6] Bruschke M V., Advani SG. A numerical approach to model non-isothermal viscous flow 
through fibrous media with free surfaces. Int J Numer Methods Fluids 1994;19:575-603. doi:10.1002/fld.1650190704.

[7] Bruschke M V, Advani SG. RTM: Filling simulation of complex three dimensional shelllike structures. Sampe Q 1991;23:2-11.

[8] Chan AW, Hwang S-T. Modeling of the impregnation process during resin transfer molding. Polym Eng Sci 1991;31:1149-56.

[9] Coulter JP, Guceri SI. Resin impregnation during the manufacturing of composite materials subject to prescribed injection rate. J Reinf Plast Compos 1988;7:200-19.

[10] García JA, Gascón L, Sánchez F, Chinesta F. Mould filling simulation in RTM processes. Reveu Des Compos Des Mater Av 2003:85-98.

[11] Gascón L, García JA, LeBel F, Ruiz E, Trochu F. A two-phase flow model to simulate mold filling and saturation in Resin Transfer Molding. Int J Mater Form 2015. doi:10.1007/s12289-015-1225-z.

[12] Liu B, Bickerton S, Advani SG. Modelling and simulation of resin transfer moulding (RTM) — gate control, venting and dry spot prediction. Compos Part A Appl Sci Manuf 1996;27:135-41.

[13] Montés N, Sánchez F. A new computational tool for liquid composite moulding process design based on configuration spaces. Compos Part A Appl Sci Manuf 2010;41:58-77. doi:10.1016/j.compositesa.2009.07.003.

[14] Sánchez F, García JA, Gascón L, Chinesta F. Towards an efficient numerical treatment of the transport problems in the resin transfer molding simulation. Comput Methods Appl Mech Eng 2007;196:2300-12. doi:10.1016/j.cma.2006.07.018.

[15] Šimáček P, Advani SG. Desirable features in mold filling simulations for liquid composite molding processes. Polym Compos 2004;25:355-67.

[16] Simacek P, Advani SG, Binetruy C. Liquid injection molding simulation (LIMS) a comprehensive tool to design, optimize and control the filling process in liquid composite molding. JEC--Composites 2004;8:58-61.

[17] Soukane S, Trochu F. Application of the level set method to the simulation of resin transfer molding. Compos Sci Technol 2006;66:1067-80.

doi:10.1016/j.compscitech.2005.03.001.

[18] Trochu F, Gauvin R, Gao D. Numerical analysis of the resin transfer molding process by the finite element method. Adv Polym Technol 1993;12:329-42.

[19] Trochu F, Ruiz E, Achim V, Soukane S. Advanced numerical simulation of liquid composite molding for process analysis and optimization. Compos Part A Appl Sci Manuf 2006;37:890-902.

[20] Yoo Y-E, Lee W Il. Numerical simulation of the resin transfer mold filling process using the boundary element method. Polym Compos 1996;17:368-74.

[21] Young WB al, Rupel K, Han K, Lee LJ, Liou MJ. Analysis of resin injection molding in molds with preplaced fiber mats. II: Numerical simulation and experiments of mold 
filling. Polym Compos 1991;12:30-8.

[22] Young WB, Fong K, Lee LJ. Flow simulation in molds with preplaced fiber mats. Polym Compos 1991;12:391-403.

[23] Bruschke M V, Advani SG. A finite element/control volume approach to mold filling in anisotropic porous media. Polym Compos 1990;11:398-405. doi:10.1002/pc.750110613.

[24] Mathur R, Advani SG, Fink BK. Use of genetic algorithms to optimize gate and vent locations for the resin transfer molding process. Polym Compos 1999;20:167-78.

[25] Gokce A, Hsiao KT, Advani SG. Branch and bound search to optimize injection gate locations in liquid composite molding processes. Compos Part A-Applied Sci Manuf 2002;33:1263-72. doi:10.1016/s1359-835x(02)00047-7.

[26] Young W-B. Gate location optimization in liquid composite molding using genetic algorithms. J Compos Mater 1994;28:1098-113.

[27] Yu H-W, Young W-B. Optimal design of process parameters for resin transfer molding. J Compos Mater 1997;31:1113-40.

[28] Kim BY, Nam GJ, Lee JW. Optimization of filling process in RTM using a genetic algorithm and experimental design method. Polym Compos 2002;23:72-86. doi:10.1002/pc. 10413 .

[29] Lin MYY, Murphy MJJ, Hahn HTT. Resin transfer molding process optimization. Compos Part A Appl Sci Manuf 2000;31:361-71. doi:10.1016/S1359-835X(99)00054-8.

[30] Jiang S, Zhang C, Wang B. Optimum arrangement of gate and vent locations for RTM process design using a mesh distance-based approach. Compos Part A Appl Sci Manuf 2002;33:471-81.

[31] Ye X, Zhang C, Liang Z, Wang B. Heuristic algorithm for determining optimal gate and vent locations for RTM process design. J Manuf Syst 2004;23:267-77.

[32] Luo J, Liang Z, Zhang C, Wang B. Optimum tooling design for resin transfer molding with virtual manufacturing and artificial intelligence. Compos Part A Appl Sci Manuf 2001;32:877-88.

[33] Jiang S, Zhang C, Wang B. A process performance index and its application to optimization of the RTM process. Polym Compos 2001;22:690-701.

[34] Du Q, Faber V, Gunzburger M. Centroidal Voronoi tessellations: applications and algorithms. SIAM Rev 1999;41:637-76.

[35] Lugo J, Simacek P, Advani SG. Analytic method to estimate multiple equivalent permeability components from a single rectilinear experiment in liquid composite molding processes. Compos Part A Appl Sci Manuf 2014;67:157-70.

[36] Wang J, Simacek P, Advani SG. Fast Mold Filling Simulation Based on the Geodesic Distance Calculation Algorithm for Liquid Composite Molding Processes. C Comput Model Eng Sci 2015;107:59-79.

[37] Du Q, Emelianenko M, Ju L. Convergence of the Lloyd algorithm for computing 
centroidal Voronoi tessellations. SIAM J Numer Anal 2006;44:102-19.

[38] Lloyd SP. Least squares quantization in PCM. Inf Theory, IEEE Trans 1982;28:129-37.

[39] Cai Z. Simplified mold filling simulation in resin transfer molding. J Compos Mater 1992;26:2606-30.

[40] Jovanovic V, Manoochehri S, Chassapis C. Parameter estimation for resin transfer molding. Eng Comput 2001;18:1091-107.

[41] Šimáček P, Advani SG. Permeability model for a woven fabric. Polym Compos 1996;17:887-99.

[42] De Parseval Y, Pillai KM, Advani SG. A simple model for the variation of permeability due to partial saturation in dual scale porous media. Transp Porous Media 1997;27:24364.

[43] Di Fratta C, Klunker F, Trochu F, Ermanni P. Characterization of Textile Permeability as a Function of Fiber Volume Content with a Single Unidirectional Injection Experiment. Compos Part A Appl Sci Manuf 2015.

[44] Bickerton S, Šimáček P, Guglielmi SE, Advani SG. Investigation of draping and its effects on the mold filling process during manufacturing of a compound curved composite part. Compos Part A Appl Sci Manuf 1997;28:801-16. 


\section{Figure Captions}

Figure 1. One-dimensional flow model with mesh

Figure 2. 1D model (equation (8)) is further generalized for weighted geodesic distance and weighted fill time for node $\mathrm{n}$ over a spatial surface

Figure 3. Control volume assignment for each node in FE/CV approach [2,13,14].

Figure 4. Voronoi Diagram of a square surface using (a) Euclidian distance; and using (b) the distance defined in equation (7)

Figure 5. Flow pattern for a square part surface when resin is introduced from four injection gates, and four Voronoi Diagrams are generated based on the fill time results.

Figure 6. Comparison between regular VD (left) and CVD (right) of the square surface with two injection gates

Figure 7. Search for the centroid of a Voronoi Diagram through running LIMS for homogeneous part: (a) Voronoi Diagram generation for three-gate injection of an orthotic foot (b) flow centroid found for $V_{1}$ by running infusion simulation by setting infusion lines on the region boundary

Figure 8. Search for the centroid of a Voronoi Diagram for non-homogeneous part: (a) low permeability section at the heel due to preform compaction; (b) centroid found for Voronoi Diagram $V_{1}$.

Figure 9. Flow chart that optimizes the location of k gates using Lloyd's algorithm

Figure 10. Use of Lloyd's algorithm to find the optimal four-gate injection plan for a square surface.

Figure 11. Different initial four-gate configurations for the square converge to the same optimal gate locations.

Figure 12. An example of four-gate location optimization process converging to the local optimum for the square geometry. 
Figure 13. Three-gate optimization for the square with anisotropic fabric: (a) optimal gate locations for $0^{\circ}$ laminates after 6 iterations (24 simulations $K_{1}=2 K_{2}$ :); (b) optimal gate locations for $-45^{\circ}$ laminates after 5 iterations (20 simulations $K_{1}=2 K_{2}$ ); (c) optimal gate locations for $0^{\circ}$ laminates after 4 iterations (16 simulations $K_{1}=10 K_{2}$ :); (d) optimal gate locations for $-45^{\circ}$ laminates after 4iterations (16 simulations $\left.K_{1}=10 K_{2}\right)$

Figure 14. Orthotic part design and optimal one-gate injection fill time result

Figure 15. Two-gate location optimization for the orthotic leg

Figure 16. Three-gate location optimization for the orthotic leg which satisfies the resin gel time restrictions

Figure 17. Two-gate injection optimization results comparison when using (a): exhaustive search on the coarse mesh with 210 nodes, (b): Genetic Algorithm after 190 simulations, and (c): CVD method after 12 simulations.

Figure 18. Three-gate injection optimization results comparison when using (a): Genetic Algorithm after 230 simulations, and (b) CVD method after 28 simulations.

Figure 19. Two gate optimization for a chassis: (a) part geometry with homogeneous isotropic permeability, (b) optimal two-gate results after 15 simulations, (c) part geometry with low permeability region, (d) optimal two-gate results after 18 simulations.

Figure 20. Two gate location optimization for the chassis with flow disturbance (high permeability) region shown in (a), and (b) VDs calculation in iteration 5, and (c) inaccurate centroid found for $V_{1}$ because of the extra flow front meeting point. 
Fig_1

$K_{i} \phi_{i} h_{i}$

Flow Front

$$
i-1
$$

$\stackrel{x_{i}}{\longrightarrow}$

Injection Gate
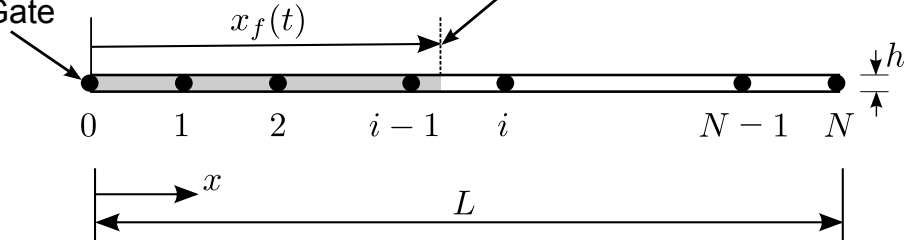


\section{Fig_2}

Resin travel path

\section{Injection Gate}

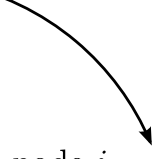

node $i$

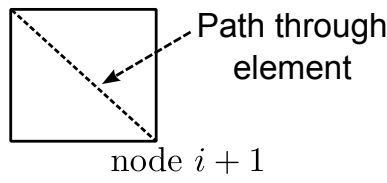

$\widetilde{\Delta x_{i}}:$ weighted distance $\widetilde{\Delta t_{i}}:$ weighted time 
- : Node

…. : Control volume boundary

: Control volume assigned to node 


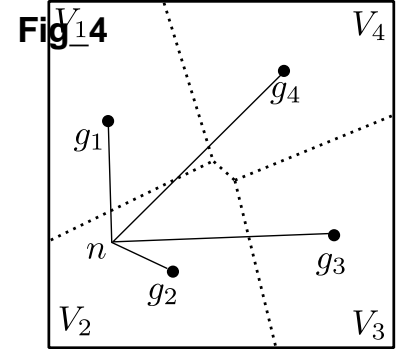

$n \in V_{2}$

$d\left(n, g_{2}\right)<d\left(n, g_{i}\right), i=1,3,4$

(a)
$V_{i}$ : Voronoi Diagram

$g_{i}:$ Generator

Voronoi Diagram Boundary

$$
\begin{aligned}
& n \in V_{2} \\
& \tilde{x}^{*^{2}}\left(n, g_{2}\right)<\tilde{x}^{* 2}\left(n, g_{i}\right), i=1,3,4
\end{aligned}
$$

(b) 


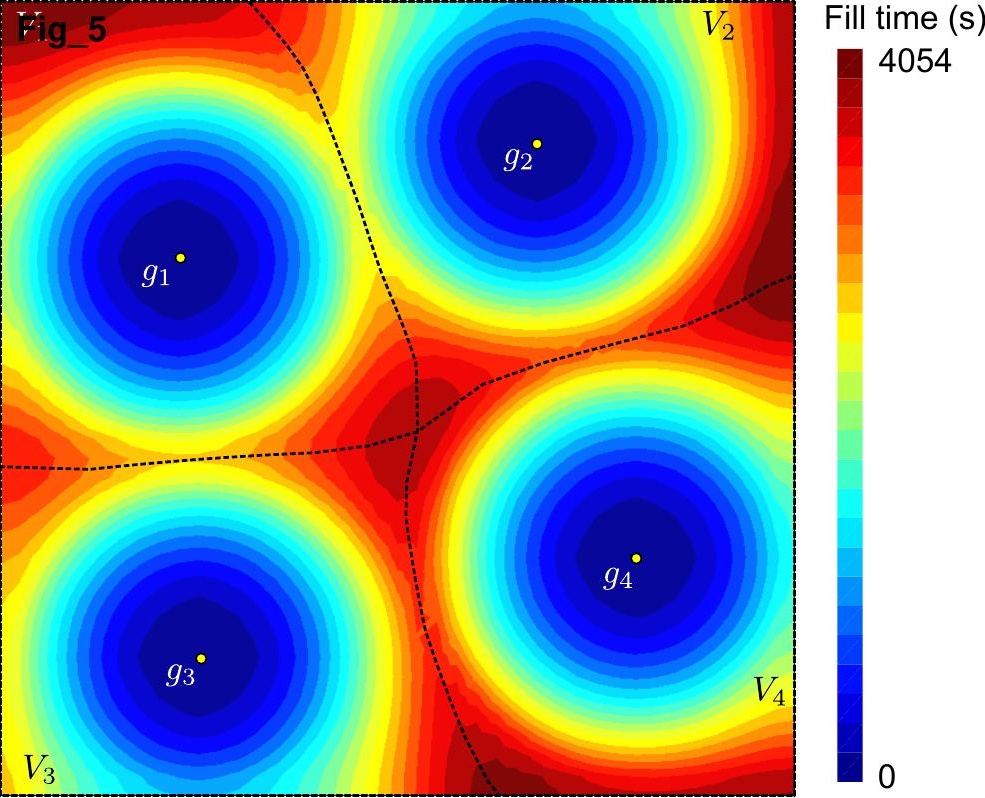



Fig_7

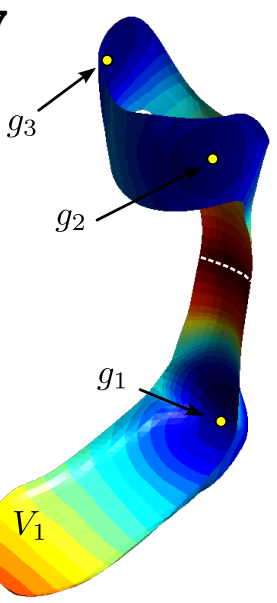

(a)

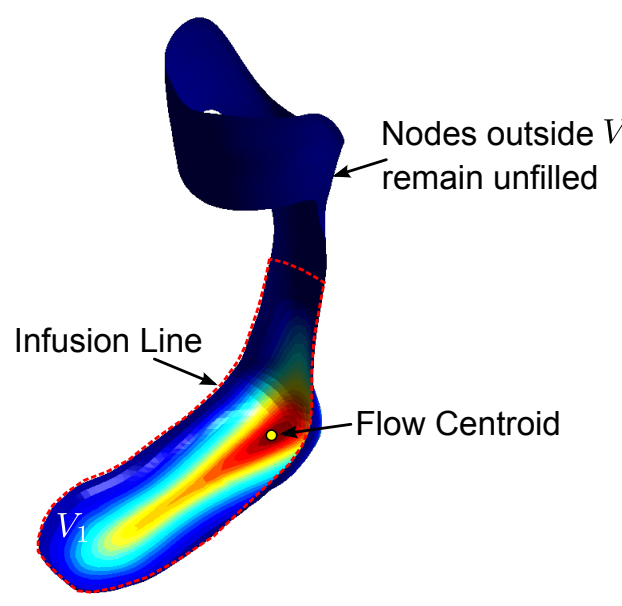

(b) 
Fig_8

Low permeability

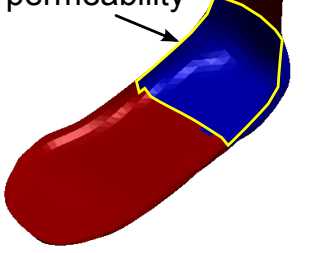

(a)

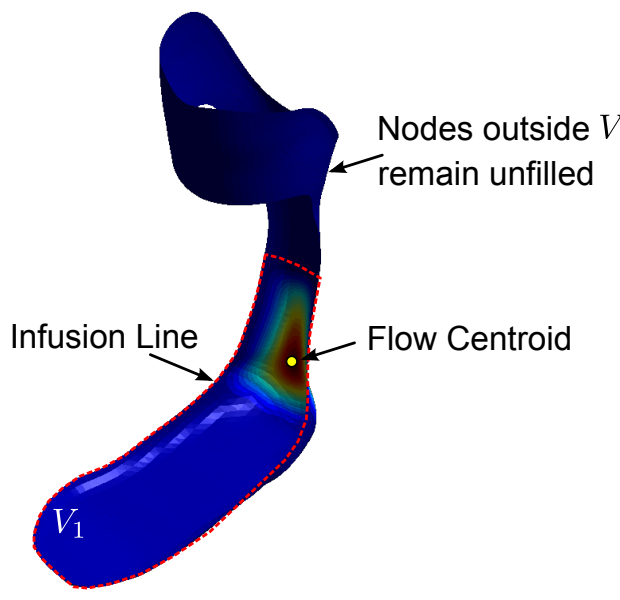

(b) 


\section{Part Mesh}

Select " $k$ " nodes randomly as gates $\left\{g_{i}\right\}(i=1, \ldots, k)$

run LIMS

(1 simulation)

Fill Time results

$\left\{g_{i}\right\}=\left\{c_{i}\right\}$

calculate VDs for $\left\{g_{i}\right\}$

$$
\left\{V_{i}\right\}(i=1, \ldots, k)
$$

calculate centroid

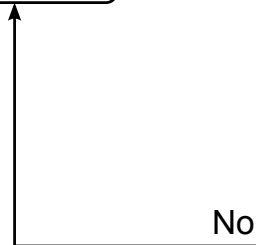

for each VD ( $k$ simulations)

$$
\left\{c_{i}\right\}(i=1, \ldots, k)
$$

No

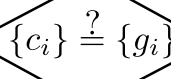

Yes

Optimal $k$-gates locations 


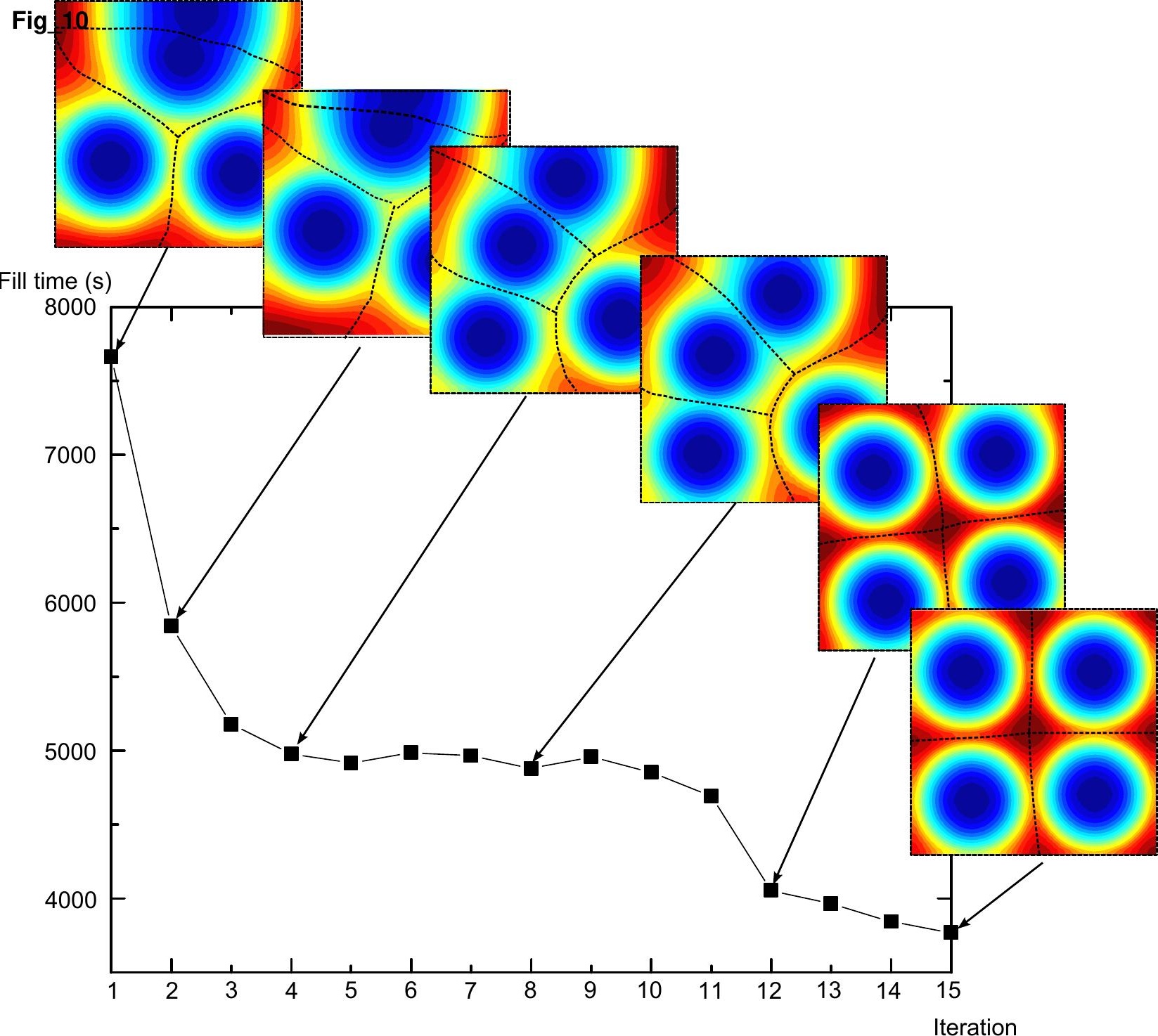


Fig_12

$g_{1}$

Fill time (s)

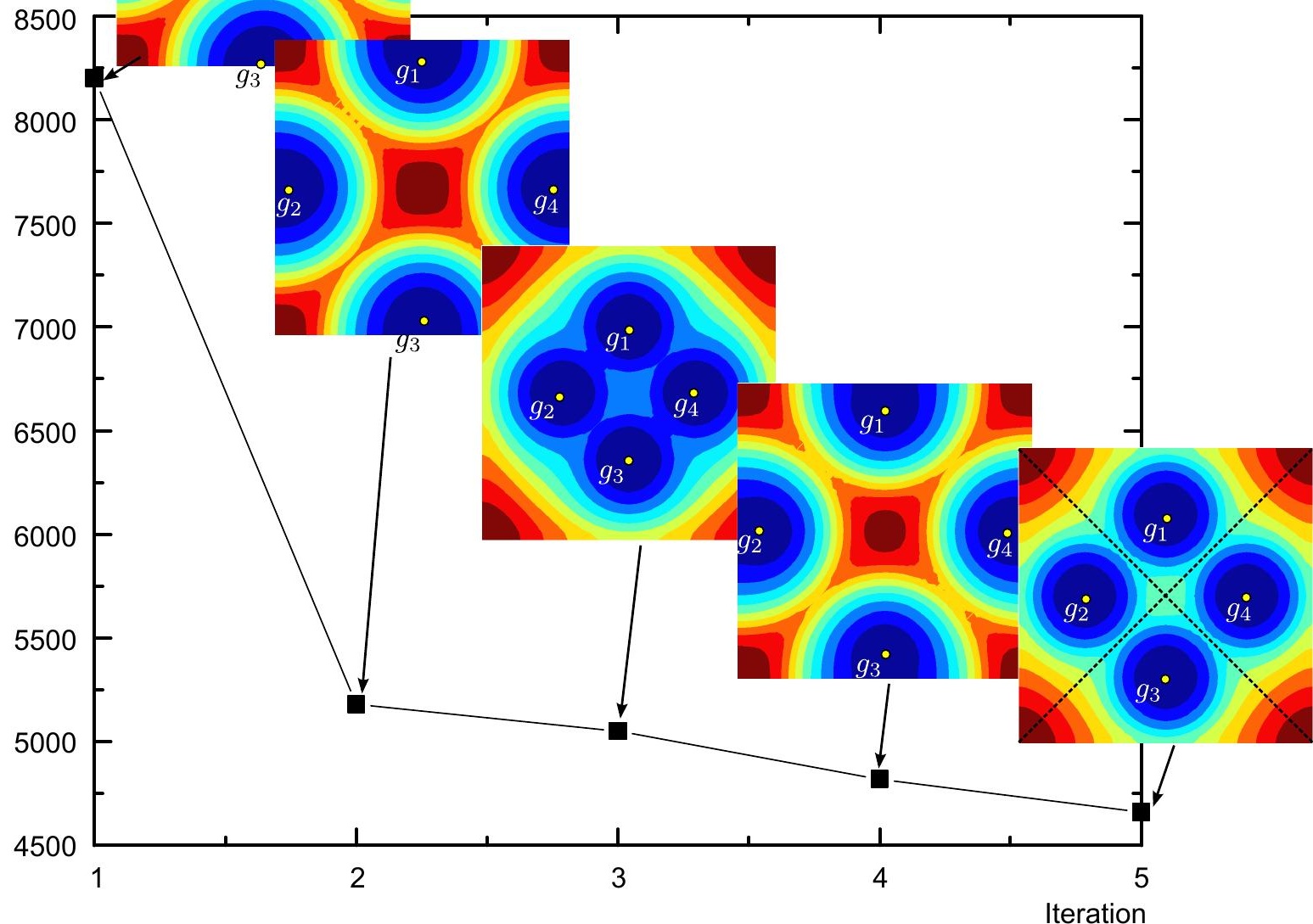




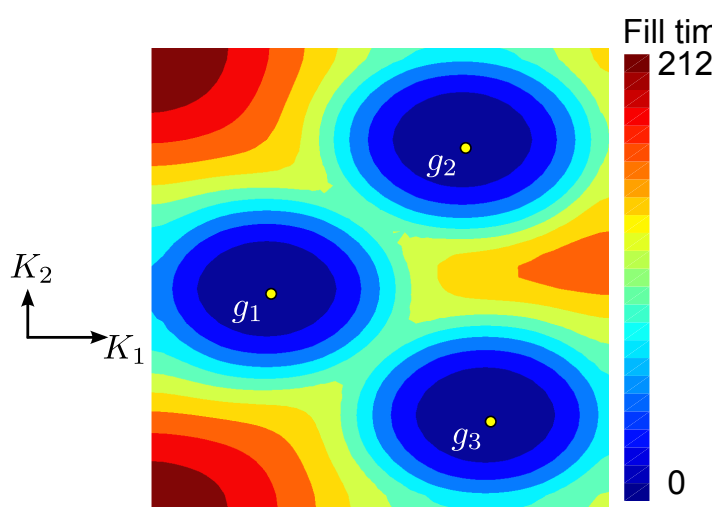

Iteration 6

(a)

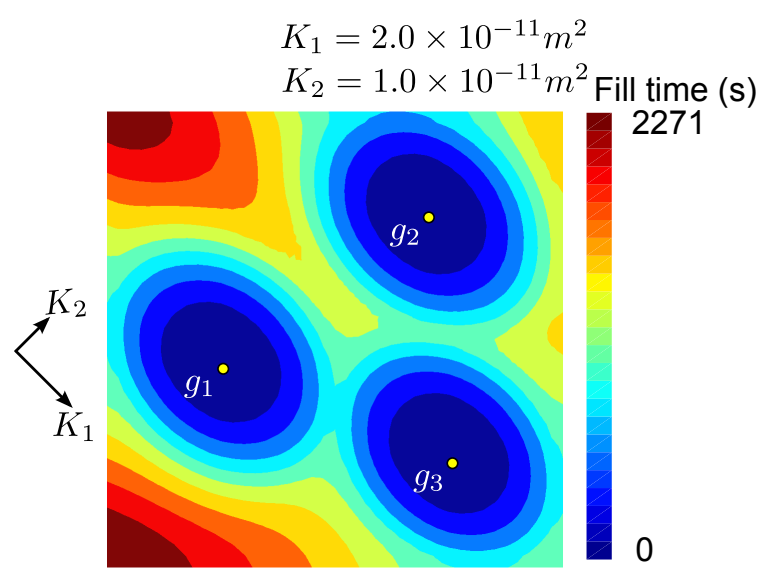

Iteration 5

(b)

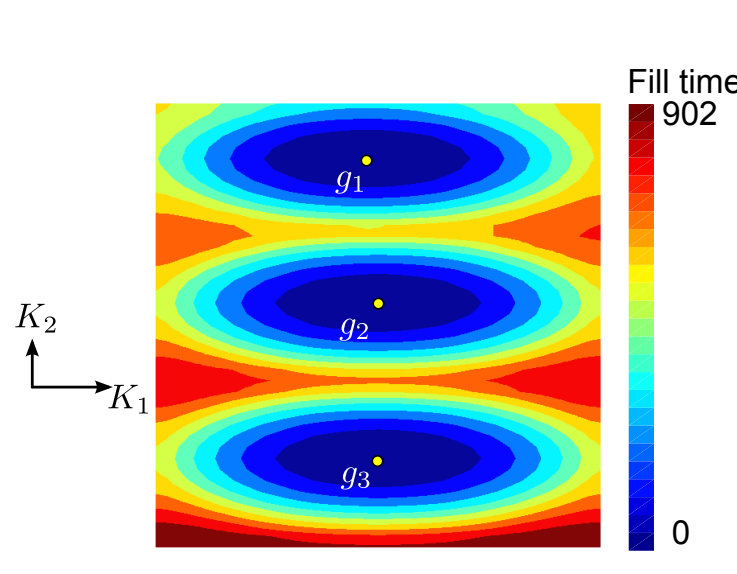

Iteration 4

(c)

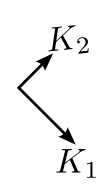

$K_{1}=1.0 \times 10^{-10} \mathrm{~m}^{2}$

$K_{2}=1.0 \times 10^{-11} \mathrm{~m}^{2}$

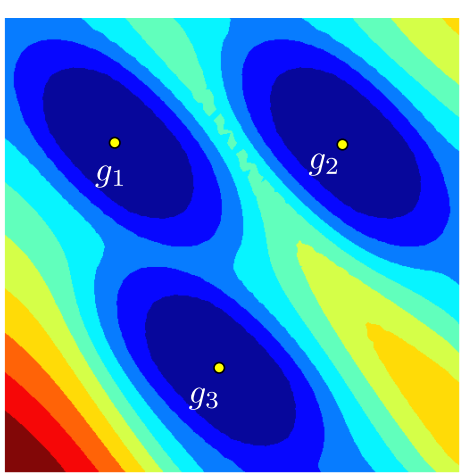

Fill time (s)

Iteration 4

(d)

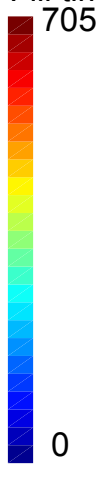


Fig_14

Resin gel time: 1200 s

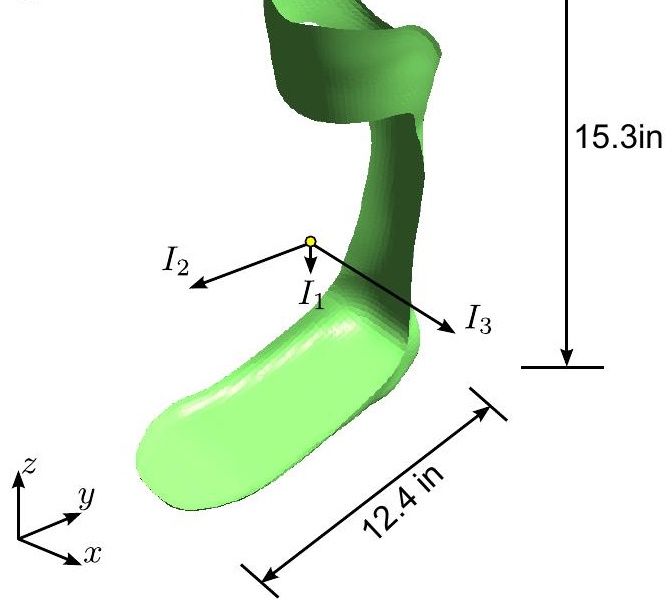

Fill Time (s) 4468
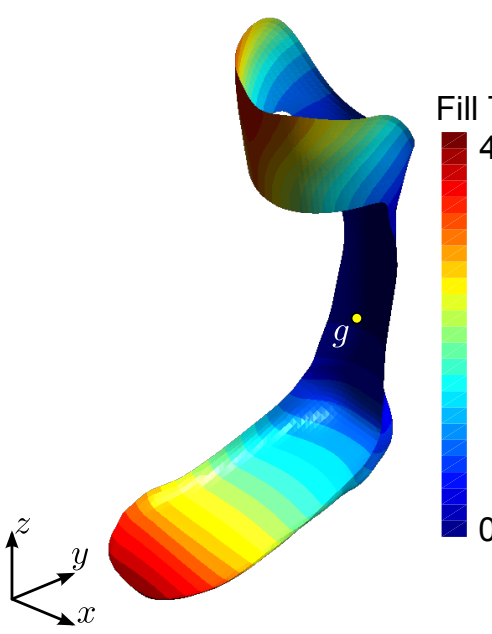
Fig_15

Fill time (s)

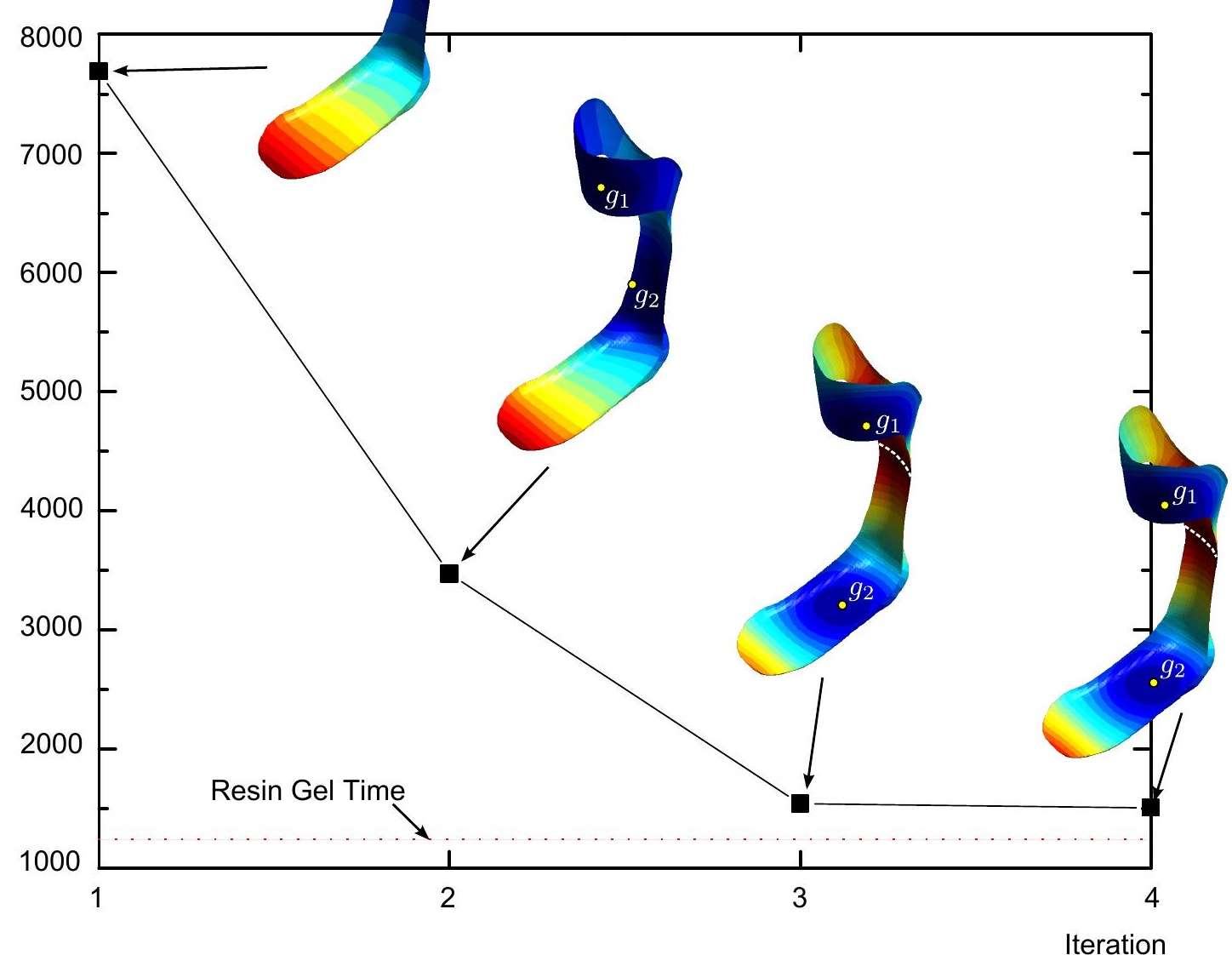


Fill ime (s)

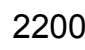

2200

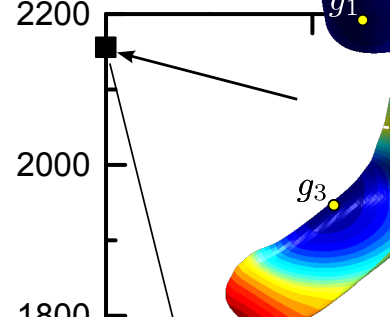

1800

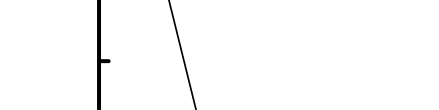

1600
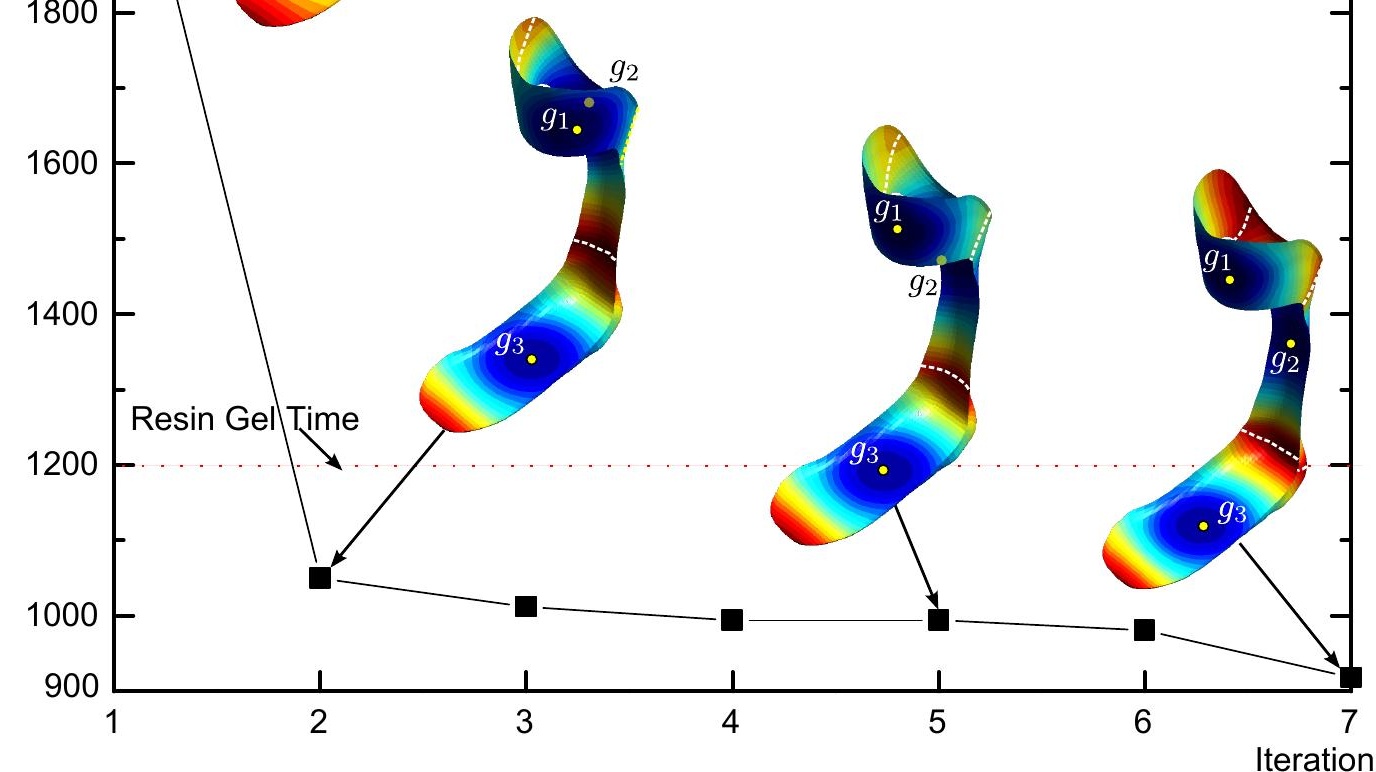

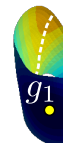

$g_{1}$

$g_{2}$

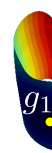

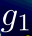
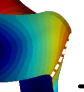

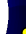


Fig_17 Fill Time (s)
1458

0

\section{(s)}

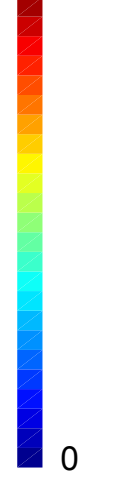

(a)
Fill Time (s)

1481

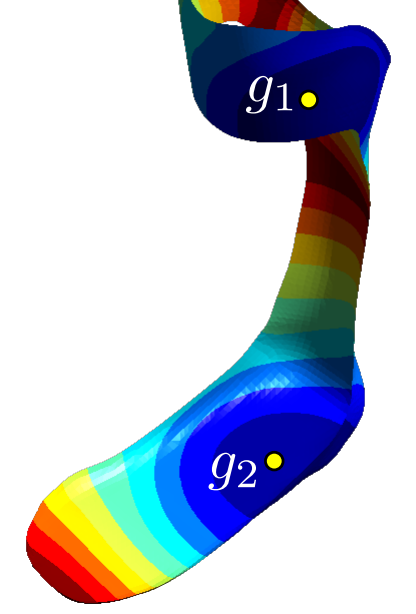

(b)

0
Fill Time (s)

1506

(c) 
Fig_18

Fill Time (s)

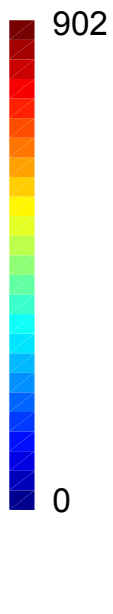

Fill Time (s)

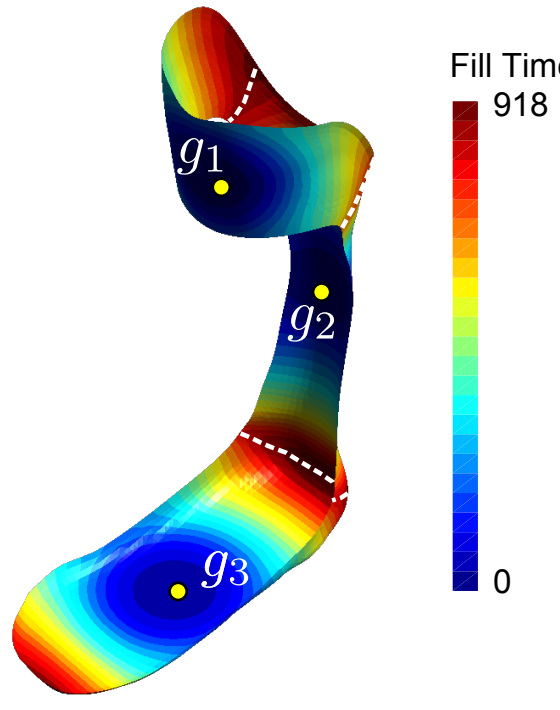

(a)

(b) 
Fill Time (s)
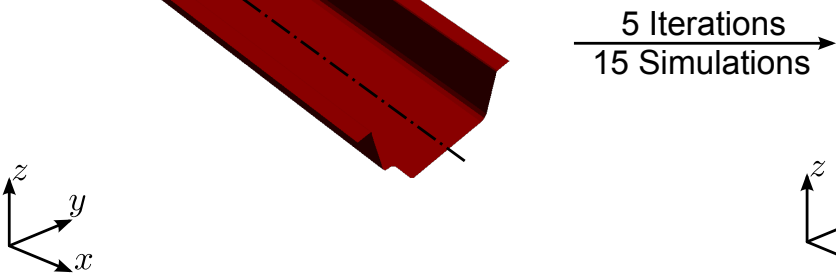

(a)

$$
K=1.9 \times 10^{-11} \mathrm{~m}^{2}
$$
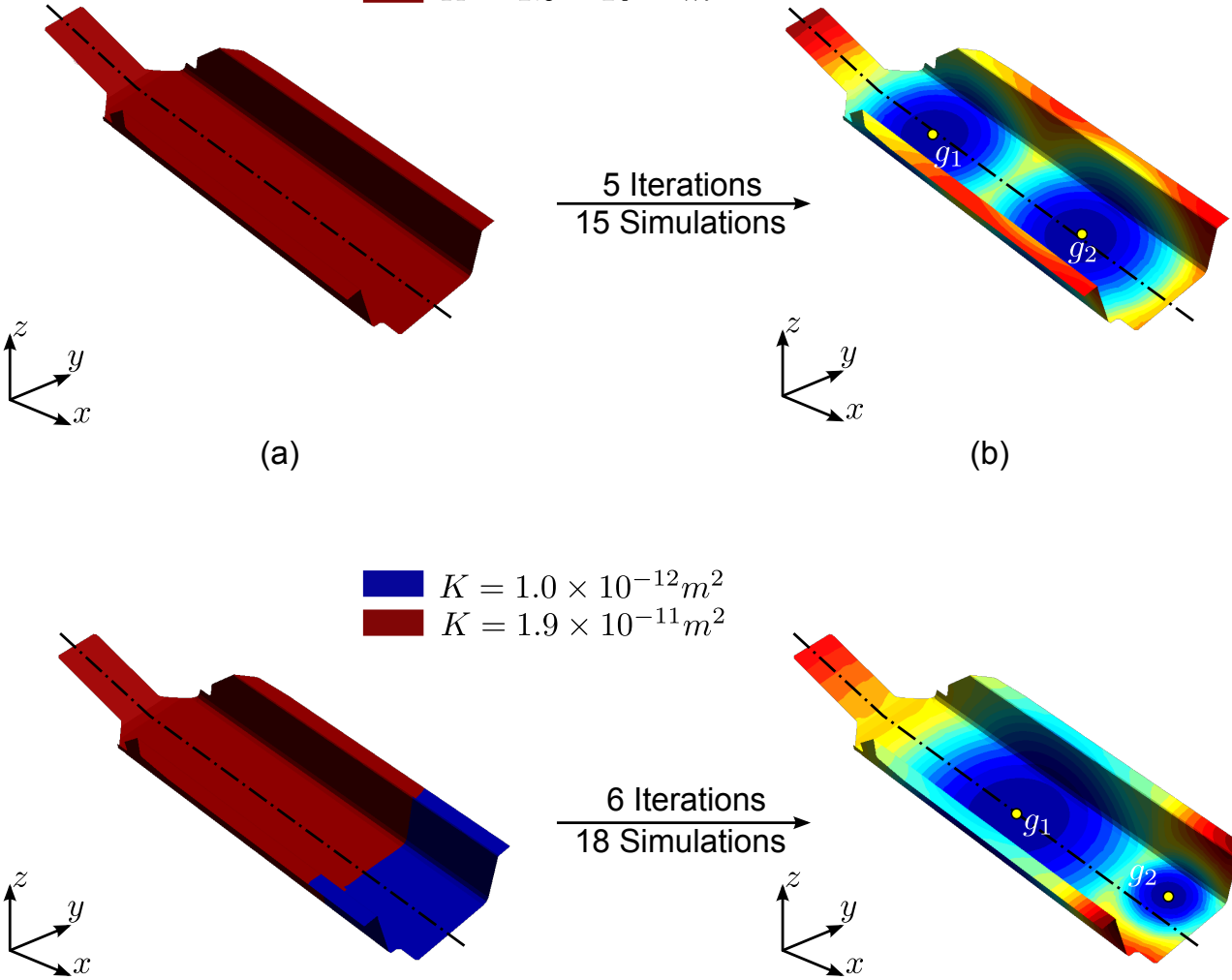

(b)
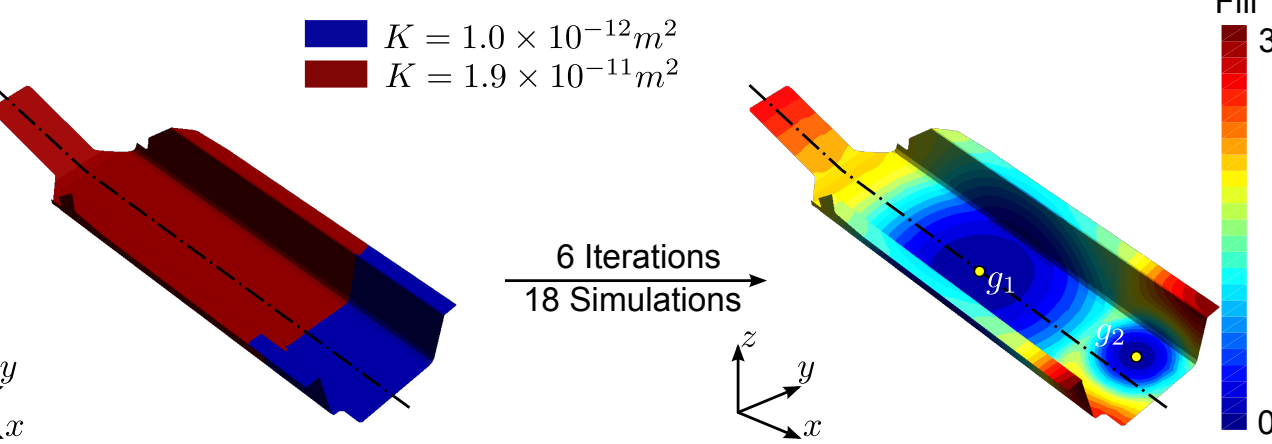

Fill Time (s)

(c)

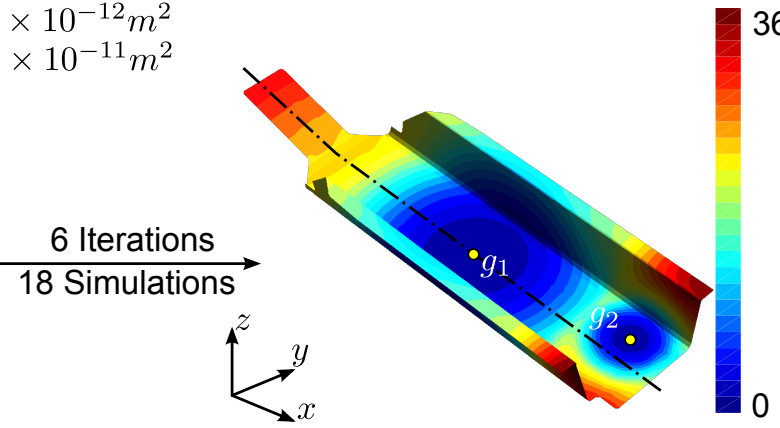
3620 


\section{Fig_20 \\ $K=1.92 \times 10^{-11} \mathrm{~m}^{2}$ \\ $K=1.0 \times 10^{-8} \mathrm{~m}^{2}$}

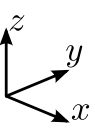

(a)

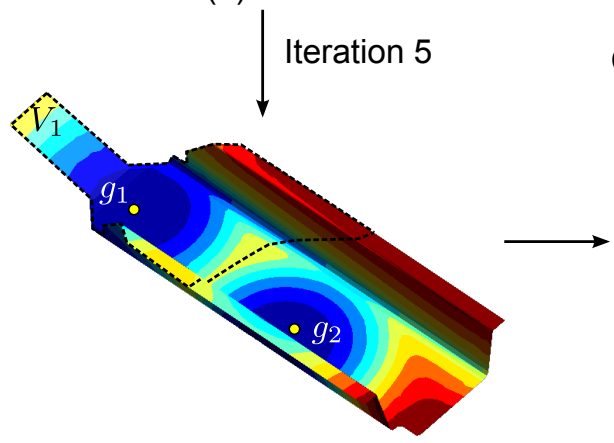

Centroid found for $V_{1}$

(b)

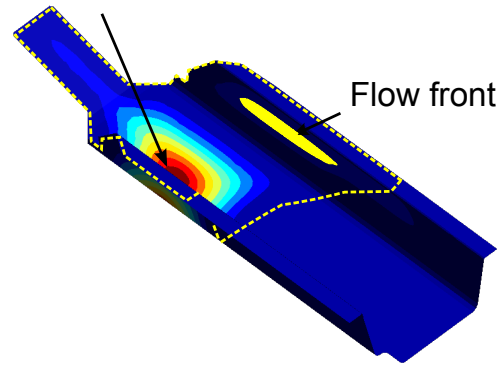

(c) 